\title{
Intrinsic and photo-induced optical properties of photoaligning azo-based materials coupled with liquid crystal systems.
}

\author{
N. Podoliak, ${ }^{1}$ E. Mavrona, ${ }^{1}$ S. Mailis, ${ }^{2}$ J.R.E.Gill, ${ }^{3}$ G. D'Alessandro,${ }^{3}$ V. Apostolopoulos, ${ }^{1}$ \\ N. Tabiryan ${ }^{4}$ and M.Kaczmarek ${ }^{1}$ \\ 1 Physics and Astronomy, University of Southampton, Southampton, SO17 1BJ, United Kingdom \\ 2 Optoelectronics Research Centre, University of Southampton, Southampton, SO17 1BJ, United Kingdom \\ 3 Mathematical Sciences, University of Southampton, Southampton, SO17 1BJ, United Kingdom \\ 4 Beam Engineering for Advanced Measurements Company, 809 South Orlando Avenue Suite I, Winter Park, \\ Florida 3278, USA
}

Recent developments in photoalignment of liquid crystals (LCS) brought to the market photoreversible materials such as azobenzene-based PAAD complex dyes [1], which quickly found broad photonics applications for constructing diffraction elements and waveplates [2,3]. Despite extensive investigations of photoaligned LC systems, there is little information on the optical properties such thin PAAD layers.

Here we report an experimental investigation of optical properties, such as refractive indices and absorption coefficients for different PAAD materials, namely PAAD-22D, 22E and 22N. Photoinduced phase gratings were recorded in 20-50 nm thick PAAD layers with no evidence of a corresponding surface relief, which is a typical feature in thicker azobenzene films. Therefore, the formation of the gratings is attributed to optically induced birefringence. The investigated materials exhibited different values of birefringence, reaching 0.025 , and significantly different temporal response to laser irradiation. Moreover, the diffracted power was observed to be very sensitive to the polarisation of the probe beam with respect to that of the pump beam.

We also studied photoinduced diffraction in PAAD-LC systems, where we observed the formation of a stable and strong diffraction pattern. The gratings can be rewritten by illumination with another light pattern or switched off by reorienting liquid crystals with a bias voltage. A diffraction efficiency of more than $5 \%$ was measured in a cell containing PAAD-22D in combination with $8 \mu \mathrm{m}$ thick LC-E7 layer. Finally, we compare the dynamics of light induced response in the PAAD layers and PAAD-LC cells and investigate their respective memory effects.

[1] Beam Engineering for Advanced Measurements Company, Winter Park, FL 32789.

[2] Vernon, J. P., et al., Opt. Express 21, 1645 (2013).

[3] Nersisyan, S. R., et al. Opt. Express 21, 8205 (2013). 